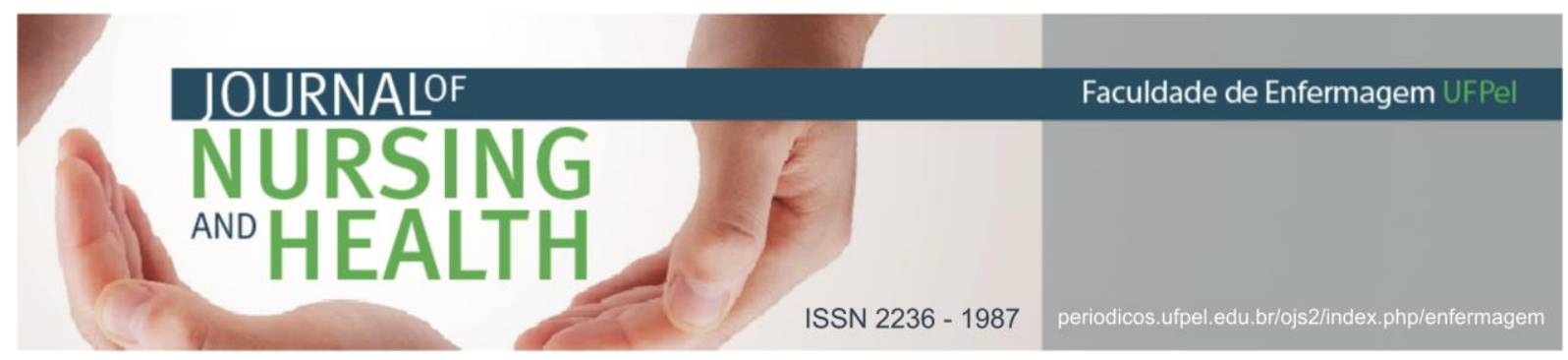

REVISÃO INTEGRATIVA

\title{
Apoio matricial na gestão do trabalho da atenção primária à saúde: uma revisão integrativa
}

\author{
Matrix support in the management of primary health care work: an integrative review \\ Apoyo matricial en la gestión del trabajo de atención primaria a la salud: revisión \\ integrativa
}

Soares, Danielle de Jesus ${ }^{\text {; }}$ Rivemales, Maria da Conceição Costa²

Como citar este artigo: Soares DJ, Rivemales MCC. Apoio matricial na gestão do trabalho da atenção primaria à saúde: uma revisão integrativa. J. nurs. health. 2019;9(2):e199207

\section{RESUMO}

Objetivo: investigar a produção bibliográfica sobre o apoio matricial na atenção primária à saúde. Métodos: trata-se de uma revisão integrativa. A busca se deu em Biblioteca Virtual de Saúde, Literatura Latino-Americana e do Caribe em Ciências da Saúde, Medical Literature Analysis and Retrieval System Online, Banco de Dados em Enfermagem, em abril a maio de 2018. A análise dos dados foi de conteúdo. Resultados: 13 artigos foram incluídos na revisão. A análise dos artigos evidenciou que o Apoio Matricial contribui para a organização do processo gerencial na Atenção Primária à Saúde e suas diferentes perspectivas e formas de apoio ofertadas; foi possível identificar também os fatores tidos como facilitadores e dificuldades para o trabalho. Considerações finais: destaca-se a importância de realizar estudos que investiguem as práticas do Apoio Matricial, seus benefícios e entraves, assim como a formação continuada dos profissionais atuantes visando à busca pela integralidade da atenção.

Descritores: Equipe de assistência ao paciente; Gestão em saúde; Atenção primária à saúde.

\begin{abstract}
Objective: to investigate bibliographic production on matrix support in primary health care. Methods: this is an integrative review. The search was done in Virtual Health Library, Latin American and Caribbean Literature in Health Sciences, Medical Literature Analysis and Retrieval System Online, Database in Nursing, in April to May of 2018. The analysis was of content. Results: 13 articles were included in the review. The analysis of the articles showed that the Matrix Support contributes to the organization of the management process in Primary Health Care and its different perspectives and forms of support offered; it was also possible to identify the factors considered as facilitators and difficulties for the work. Final considerations: it's important to carry out studies that investigate the practices of the Matrix Support, its benefits and obstacles, as well as the ongoing training of working professionals aiming at the search for integrality of care.

Keywords: Patient care team; Health management; Primary health care.
\end{abstract}

\footnotetext{
1 Enfermeira. Especialista em Clínica da Pessoa e da Família. Universidade Federal da Bahia (UFBA). E-mail: dany.sol22@hotmail.com http: / / orcid.org/0000-0003-0739-2812

2 Enfermeira. Doutora em Enfermagem. Universidade Federal do Recôncavo da Bahia (UFRB). E-mail: mariarivemales@ufrb.edu.br http://orcid.org/0000-0001-7773-4772
} 


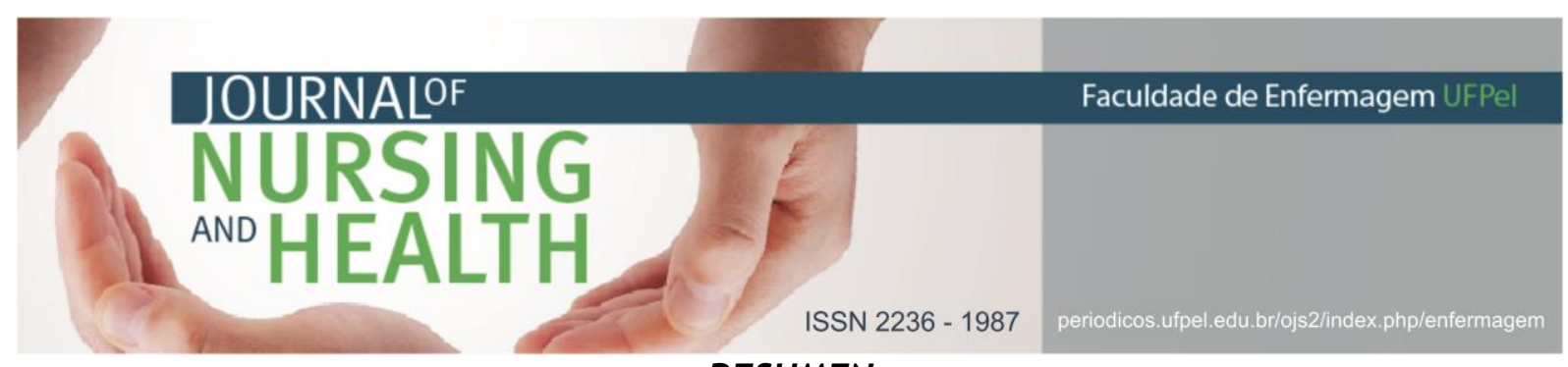

RESUMEN

Objetivo: investigar la producción bibliográfica sobre el apoyo matricial en la atención primaria a la salud. Métodos: revisión integrativa en Biblioteca Virtual de Salud, Literatura Latinoamericana y del Caribe en Ciencias de la Salud, Medical Literature Analysis and Retrieval System Online, Banco de Datos en Enfermería, en abril a mayo de 2018. El análisis fue de contenido. Resultados: 13 artículos fueron incluidos en la revisión. El análisis de los artículos evidenció que el Apoyo Matricial contribuye a la organización del proceso gerencial en la Atención Primaria a la Salud y sus diferentes perspectivas y formas de apoyo ofrecido; fue posible identificar también los factores tenidos como facilitadores y dificultades para el trabajo. Consideraciones finales: se destaca la importancia de estudios que investiguen las prácticas del Apoyo Matricial, sus beneficios y trabas, así como la formación continuada de los profesionales visando la búsqueda por la integralidad de la atención.

Descriptores: Grupo de atención al paciente; Gestión en salud; Atención primaria de salud.

\section{INTRODUÇÃO}

O Apoio Matricial (AM) surge como forma de organização da gestão nos serviços de saúde, visando superar a gerência caracterizada pela verticalização, compartimentalização e produção de trabalho fragmentado e alienador para o trabalhador. ${ }^{1}$ É uma metodologia de gestão do cuidado que foi primeiramente adotada em serviços de saúde mental no estado de Minas Gerais, Belo Horizonte, e na Argentina. Foi também incorporado, em 2003, aos programas do Ministério da Saúde como o Humaniza do Sistema Único de Saúde (SUS), Saúde Mental e Atenção Básica/Saúde da Família. $^{2}$ AM e a equipe de referência se constituem em arranjos organizacionais e metodologia de ação. As equipes de referência, que tem vínculo e responsabilização direta com os usuários, compartilham saberes, competências, responsabilidades e ações com as equipes de $A M$, assim recebem suporte de profissionais das áreas especializadas, as quais não participam cotidianamente da equipe de referência, mas ofertam suporte técnico especializado às equipes de referência. ${ }^{3}$
O AM visa constituir equipes multiprofissionais para o enfretamento de problemas, porém sem perder a responsabilização e compromisso com a produção de saúde. ${ }^{3}$ Através deste, pode-se fortalecer o compromisso profissional com saúde e condução de casos. A ação de reduzir o número de encaminhamentos, gera o fortalecimento da corresponsabilização pelos casos atendidos que visa excitar o vínculo entre equipes e entre equipes $e$ usuários. ${ }^{4}$

A troca entre os diferentes conhecimentos dos núcleos profissionais é vista como a saída para a superar o processo de trabalho individualizado e fragmentado. Nesse sentido, o caráter técnico-pedagógico do $A M$ se refere à transformação do cuidado produzido pelos profissionais com os usuários. ${ }^{4}$

Com o objetivo de ampliar a abrangência e escopo das ações ofertadas na atenção básica, bem como na sua resolubilidade, 0 Ministério da Saúde cria através da Portaria $n^{\circ} 154$, de 24 de janeiro de 2008, os Núcleos de Apoio a Saúde da Família (NASF). Diferentes 


\section{JOURNALF

profissionais podem fazer parte da equipe do NASF, estes deverão atuar com forma conjunta com os profissionais das Equipes de Saúde da Família (ESF), compartilhando práticas em saúde, atuando diretamente no apoio às equipes. ${ }^{5} \mathrm{Em} \mathrm{2017}$, através da Portaria $n^{\circ} 2.436$ de 21 de setembro de 2017, os NASF tiveram modificações no que diz respeito a sua cobertura e passaram a ser denominados de Núcleo Ampliado de Saúde da Família e Atenção Básica (NASF-AB).

Outra forma de AM para as ESF diz respeito à articulação com a saúde mental. Por meio do $A M$, a saúde mental pode ser descentralizada na Atenção Primária à Saúde (APS), configurando como uma ferramenta para o trabalho; articula-se ainda com os princípios do SUS ao contemplar o acesso universal, a integralidade e a equidade. 6

$\begin{array}{ccc}0 & \text { AM } & \text { também } \\ \text { construção } & \text { de } & \text { possibilita } \\ \text { fazeres } & \text { a }\end{array}$
conhecimentos, assim como auxilia na incorporação da prática clínica focada na integralidade do sujeito. ${ }^{7}$

Visando a qualificação do cuidado em saúde, o AM vem como reorganizador do cuidado e da gestão de casos, condutas e serviços. Apoia as equipes de referência no desempenho de suas funções e integração dos diferentes olhares, diante do ser que recebe a atenção, enriquecendo 0 fazer em saúde, assim como o arcabouço técnico-científico necessário à prática cotidiana.

Diante do exposto, nota-se a importância em aprofundar os conhecimentos sobre o tema, uma vez que o mesmo é pouco abordado na literatura nacional; além de ser fundamental para o desempenho do trabalho, baseado nos princípios e diretrizes do SUS e fundamentos da APS.

Nesse sentido, o presente estudo pretende responder a seguinte questão: Como o AM relaciona-se com a gestão do trabalho na Atenção Primária à Saúde? A fim de responder à pergunta norteadora traçou-se 0 seguinte objetivo: investigar a produção bibliográfica sobre o apoio matricial na Atenção Primária à Saúde.

\section{MATÉRIAS E MÉTODOS}

Trata-se de uma revisão integrativa, a qual agrupa resultados de estudos desenvolvidos, o que permite a síntese de resultados sem ferir a filiação epistemológica dos estudos empíricos incluídos. ${ }^{8}$ Para a sua realização, alguns passos foram seguidos, a saber: escolha da temática, delimitação do problema e objetivos da pesquisa, seleção, análise e discussão dos artigos. ${ }^{8}$ Os artigos foram selecionados através da Biblioteca Virtual em Saúde (BVS), nas bases de dados Literatura Latino-Americana e do Caribe em Ciências da Saúde (LILACS), Medical Literature Analysis and Retrieval System Online (MEDLINE), Banco de Dados em Enfermagem (BDENF), no período de abril a maio de 2018.

Foram selecionados os seguintes descritores com base nos Descritores em Ciências da Saúde (DeCS): apoio matricial, gestão em saúde, Estratégia Saúde da Família e Atenção Primária à Saúde. Adotou-se a seguinte estratégia de pesquisa: Apoio Matricial AND (gestão em saúde OR Estratégia Saúde 
da Família) e Apoio Matricial AND Atenção Primária à Saúde.

Os critérios de inclusão dos artigos foram: estar alocados nas bases de dados; textos completos em formato de artigo e disponíveis na íntegra gratuitamente; publicados nos últimos cinco anos; escritos em língua portuguesa, inglesa ou espanhola; abordar a temática desse estudo no âmbito brasileiro. Como critérios de exclusão adotou-se: estudos duplicados, publicações que não respondam aos objetivos desse trabalho e revisões de literatura.

Após seleção inicial dos artigos passou-se a etapa de avaliação crítica dos estudos com base em leitura aprofundada dos mesmos e categorização das informações para análise, discussão e apresentação dos resultados. Foi utilizada a planilha do Excel a fim de separá-los e categorizálos de acordo com a ideia central de cada estudo. Os artigos foram analisados com base na análise de conteúdo de Bardin. ${ }^{9}$

\section{RESULTADOS E DISCUSSÃO}

Após a busca, considerando os critérios de inclusão dos estudos, o número de artigos selecionados foi de 84; destes empregando-se a leitura de títulos e resumos foram excluídos 56 estudos, sendo que os duplicados foram excluídos no momento da leitura dos resumos, restando 28 artigos para leitura crítica. Destes, após leitura na íntegra buscando identificar aspectos relacionados aos objetivos desta revisão, 13 artigos foram escolhidos. 0 fluxo descrito na Figura 1, detalha as etapas da seleção dos artigos para a revisão.

A fim de melhor compreender as principais informações dos artigos incluídos nesta revisão, na Figura 2 é descrito título, ano de publicação, autores.

Figura 1: Fluxo do processo de seleção dos estudos. Santo Antônio de Jesus/BA, 2018.

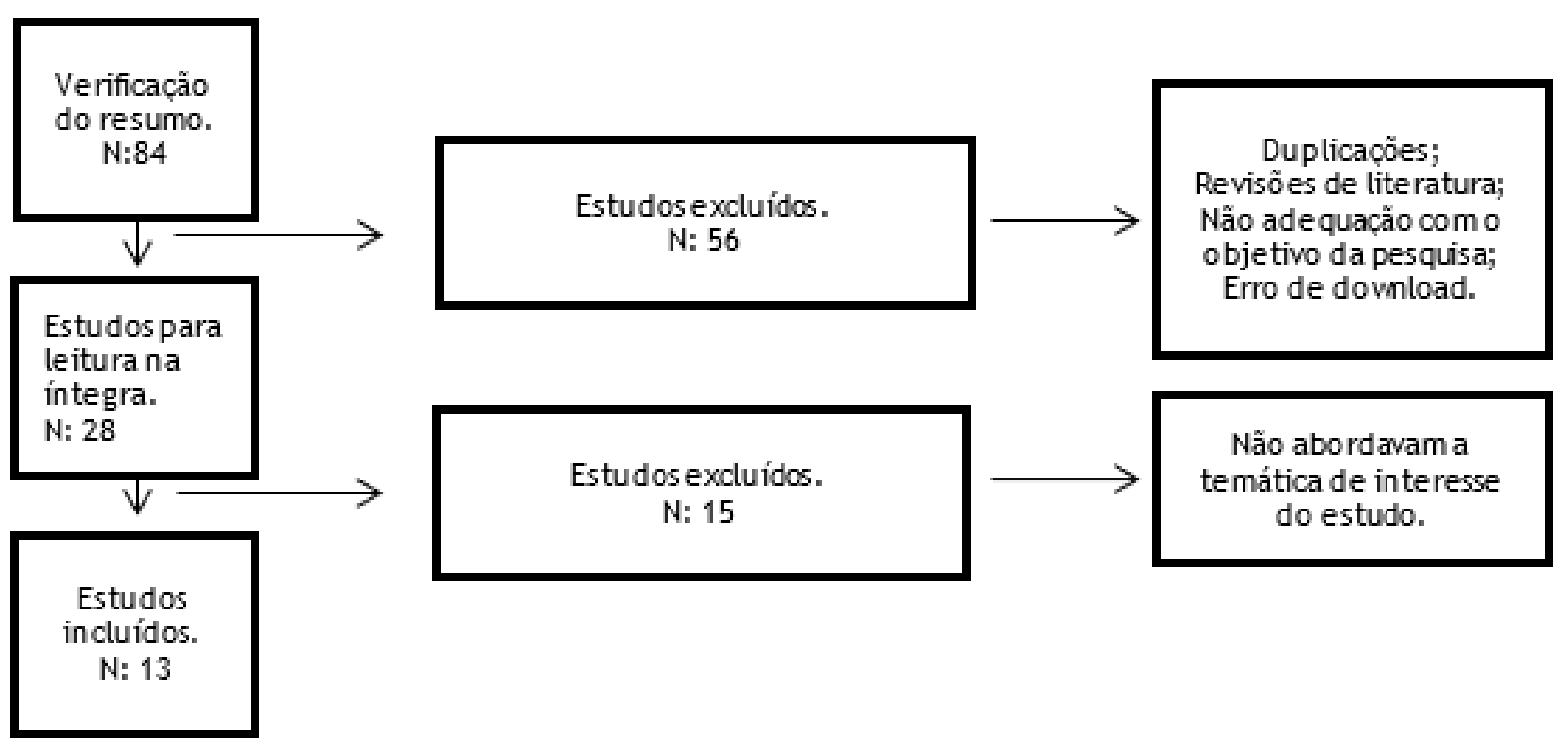

Fonte: Própria autoria. 2018. 
Figura 2:Descrição dos artigos localizados na base de dados. Santo Antônio de Jesus/BA, 2018.

\begin{tabular}{|l|l|c|l|}
\hline N. & \multicolumn{1}{|c|}{ Título do artigo } & Ano & \multicolumn{1}{|c|}{ Autores } \\
\hline 1 & $\begin{array}{l}\text { Acessibilidade e resolubilidade da assistência em saúde } \\
\text { mental: a experiência do apoio matricial }\end{array}$ & 2013 & Quinderé PHD et al \\
\hline 2 & $\begin{array}{l}\text { Núcleo de Apoio à Saúde da Família: refletindo sobre as } \\
\text { acepções emergentes da prática }\end{array}$ & 2013 & Sampaio J et al. \\
\hline 3 & $\begin{array}{l}\text { O Psicólogo apoiador matricial: percepções e práticas na } \\
\text { atenção básica }\end{array}$ & 2013 & Freire FMS, Pichelli AAWS \\
\hline 4 & $\begin{array}{l}\text { Apoio matricial em saúde mental na percepção dos } \\
\text { profissionais especialistas }\end{array}$ & 2014 & $\begin{array}{l}\text { Gerhardt Neto MR, } \\
\text { Medina TSS, Hirdes A }\end{array}$ \\
\hline 5 & $\begin{array}{l}\text { Programa de apoio matricial em cardiologia: qualificação } \\
\text { e diálogo com profissionais da atenção primária }\end{array}$ & 2014 & Hoepfner C et al. \\
\hline 6 & $\begin{array}{l}\text { A perspectiva dos profissionais da Atenção Primária à } \\
\text { Saúde sobre o apoio matricial em saúde mental }\end{array}$ & 2015 & Hirdes A. \\
\hline 7 & $\begin{array}{l}\text { Colaboração interprofissional na Estratégia Saúde da } \\
\text { Família: implicações para a produção do cuidado e a } \\
\text { gestão do trabalho }\end{array}$ & 2015 & Matuda CG et al. \\
\hline 8 & $\begin{array}{l}\text { Nas entrelinhas do olhar: apoio matricial e os profissionais } \\
\text { da Estratégia Saúde da Família }\end{array}$ & 2015 & $\begin{array}{l}\text { Fittipaldi ALM, Romano } \\
\text { VF, Barros DC. }\end{array}$ \\
\hline 9 & $\begin{array}{l}\text { Processos de trabalho dos núcleos de atenção à saúde da } \\
\text { família junto a atenção básica: implicações para a } \\
\text { Articulação de Redes Territoriais de Cuidados em Saúde }\end{array}$ & 2015 & Sampaio J et al. \\
\hline 10 & $\begin{array}{l}\text { As contribuições dos psicólogos para o matriciamento em } \\
\text { saúde mental }\end{array}$ & 2016 & Iglesias A, Avellar LZ \\
\hline 11 & $\begin{array}{l}\text { Atenção primária e saúde mental: contribuições e } \\
\text { potencialidades do apoio matricial }\end{array}$ & 2016 & Tatmatsu DB, Araújo ACC \\
\hline 12 & $\begin{array}{l}\text { Apoio matricial na atenção à saúde mental em uma } \\
\text { regional de saúde, Paraná, Brasil }\end{array}$ & 2017 & Garcia GDV et al. \\
\hline 13 & $\begin{array}{l}\text { O “cabo de força" da assistência: concepção e prática de } \\
\text { psicólogos sobre o apoio matricial no Núcleo de Apoio à } \\
\text { Saúde da Família }\end{array}$ & 2017 & Klein AP, D’Oliveira AFPL \\
\hline Fon Própria autora, 2018 & & \\
\hline
\end{tabular}

Fonte: Própria autoria, 2018.

A partir da análise dos artigos descritos na Figura 2, verificou-se que o maior percentual de publicações esteve no ano de 2015 com quatro $(30,76 \%)$, seguido do ano de $2013 \mathrm{com}$ três publicações $(23,07 \%)$, enquanto dos anos de 2014, 2016 e 2017 foram encontradas duas publicações cada $(15,38 \%)$.

A maioria dos estudos correspondendo a 8 artigos, traziam resultados de pesquisas relacionadas ao AM ofertado no campo da saúde mental, enquanto os demais ou seja, 4 artigos, abordavam o AM de forma mais ampla na ESF, enquanto um artigo trouxe os resultados referentes a implantação do AM no campo da cardiologia na APS. Os resultados apontaram para o predomínio de estudos sobre $A M$ na área da saúde mental.

A análise dos estudos evidenciou as diferentes perspectivas e formas de apoio ofertado através do matriciamento; foi possível identificar também os fatores tidos como facilitadores e dificultadores do trabalho, assim como correlacionar o 
ISSN 2236 - 1987

processo de trabalho do AM com a gestão do trabalho na APS. Dessa maneira, buscando melhor visualização e discussão dos resultados de acordo com os objetivos desta revisão, os mesmos foram divididos em duas categorias que serão esplanadas a seguir:

Apoio matricial e gestão do cuidado na Atenção Primária à Saúde

Os estudos demonstraram as principais formas de apoio ofertados através do matriciamento às equipes de APS, são elas: o atendimento em conjunto; discussão de casos; avaliação e orientação de casos; identificação de casos tratados de forma errônea; construção de Projetos Terapêutico Singular (PTS); reuniões intersetoriais; visitas domiciliares; troca de saber; capacitação e supervisão.

Alguns profissionais relataram preferência por atendimentos em conjunto, enquanto outros priorizaram as discussões de casos clínicos, no entanto o atendimento em conjunto foi classificado como enriquecedor para o trabalho, sendo a modalidade preferida tanto pelos que o executam quanto pelos que vivenciam outras formas de matriciamento; porém esta irá depender dos arranjos feitos entre equipes de apoiadores e de referência. ${ }^{6,10-11}$

Foi destacada em alguns estudos a realização de atividades assistencialformativa, técnico-burocrático, técnico-pedagógico, atividades intersetoriais, apoio políticoinstitucional, monitoramento de indicadores, discussão de protocolos e organização de ações estratégicas como campanhas, além de atividades de cunho burocráticas e administrativas que geravam a ideia do apoiador com coordenador-gerente do serviço, causando distanciamento na relação com os demais profissionais. ${ }^{12-}$ 13 Os autores chamam atenção para a possibilidade de produção de novos conhecimentos ao atuar na gestão coletiva dos processos de cuidado, articulando os diferentes segmentos, o que por sua vez favorece a reorientação do processo de trabalho em equipe interdisciplinar. ${ }^{13}$

Foi percebido nos estudos que as diferentes modalidades de apoio ofertado podem causar controversas no entendimento do real papel do $A M$, para além de poder gerar dúvidas quanto à principal atribuição desta função. Ademais é notória a necessidade de afinamento da proposta de acordo com a realidade vivenciada por cada equipe de profissionais, das equipes de referência, assim como dos apoiadores.

O AM possibilita troca de saber, supervisão e capacitação das equipes de saúde; essa troca foi percebida como via de mão dupla, em que apoiadores aprendiam com a equipe de referência e vice-versa, enriquecendo o trabalho conjunto. ${ }^{10,14} \mathrm{~A}$ troca de saber, proporcionada pelo $A M$, possibilita a ampliação dos olhares sobre as diferentes situações de trabalho, assim como as possibilidades de resolução do caso.

Outras ações destacadas referemse à identificação de casos antes negligenciados ou diagnosticados de forma errônea, manejo de casos 
ISSN 2236 - 1987

considerados mais complexos e possibilidade de escuta do sofrimento, evitando a prática contínua da medicalização. ${ }^{6,12}$

Alguns estudos ${ }^{12,15}$ ressaltam as reuniões realizadas entre equipes de apoio e equipe de saúde da família como uma forma de matriciamento, um recurso para o planejamento das ações; porém alguns entrevistados 0 consideraram como perda de tempo, uma vez que o encaminhamento para o serviço especializado poderia ser feito como forma de solucionar o caso.

Esse fato remete a reflexão sobre os reais entendimentos por parte dos profissionais sobre as potencialidades e objetivos do matriciamento e sobre a corresponsabilização de casos na atenção básica.

O acionamento da rede de serviços pela equipe de $A M$ foi mencionado em alguns estudos ${ }^{11,16-17}$ evidenciando novas formas de articulação entre os diferentes níveis de assistência, efetivação do trabalho, favorecimento da circulação dos usuários nos diferentes pontos, além da organização de fluxos de demanda.

Porém em outro estudo foi criticada a função de acionamento da rede centrada na figura do apoiador, uma vez que restringiu o acesso aos serviços especializados pelos membros da equipe de referência, comprometendo a autonomia destes últimos. ${ }^{17} \mathrm{Em}$ contrapartida foi destacada que a aproximação com especialistas facilita o agendamento de consultas e encaminhamentos, porém pontua que essa relação se limitada a estes aspectos pode dificultar o acesso aos serviços. ${ }^{18}$
Reavaliação

periódica de encaminhamentos foi verificado através do auxílio do apoiador profissional especializado. Essa ação refletiu positivamente na redução da fila para especialista, contribuindo para a gestão da mesma. Ainda no referido estudo, os entrevistados notaram que a função do $A M$ é maior que a simples discussão de casos e redução das filas para especialista, uma vez que gerou a qualificação de encaminhamentos e dos profissionais para o enfrentamento de problemas estruturais. ${ }^{19}$

0 aumento da resolutividade na atenção básica também foi percebido como função do $A M$; a corresponsabilidade pelo cuidado prestado à população também foi destacada no leque de ações do matriciamento. ${ }^{20}$

Nesse sentido, as diferentes formas de AM trouxeram, em maior ou menor intensidade, alternativas para melhoria da gestão do cuidado nas equipes de saúde da família, seja relacionado à qualificação do trabalho desenvolvido, aquisição de conhecimentos com consequente identificação e estratificação de casos com maior segurança, redução de filas para especialidades, articulação em rede, discussão de casos e consultas compartilhadas.

\section{Facilidades e dificuldades do Apoio Matricial}

Os estudos ratificam alguns fatores facilitadores do AM, são eles: aumento da corresponsabilização, diminuição de encaminhamentos desnecessários, manejo de casos mais complexos, intercâmbio com serviços 


\section{JOURNALOF \\ NURSING \\ AND HEALTH}

da rede, favorecimento no acesso aos serviços de saúde. ${ }^{6,11}$

0 vínculo com o profissional que realiza $O$ AM facilita 0 trabalho intersetorial e interdisciplinar. ${ }^{6}$ Estudo que trata sobre o $A M$, em cardiologia, destacou que é mister o envolvimento da equipe na implantação e definição de formas de ação e estratégias, de modo a possibilitar maior compreensão e aceitação dos profissionais envolvidos no cuidado. ${ }^{19}$

Contudo, alguns estudos evidenciaram fatores considerados como dificultadores para o processo de matriciamento na APS. Dentre os fatores relacionados aos profissionais de saúde, destacam-se a falta de conhecimento e/ou diferentes entendimentos sobre $O A M$, desinteresse profissional, resistência profissional na adoção da metodologia, pouca utilização e/ou compreensão do potencial do apoio. ${ }^{6,10-11,14,21}$

Quanto aos fatores relacionados aos serviços, os estudos destacam: a dificuldade de encaminhamento por profissional não apoiador, rotatividade profissional da ESF, pouco tempo de implantação das equipes, excesso de atribuições na ESF e falta de carga horária. 6,14, 20-21

Estudos destacam também a fragmentação do sistema; falta de estrutura, recursos financeiros, humanos e políticos como barreiras para a execução do $A M .^{10-11} \mathrm{O} A M$ foi descrito como cobrança, hierarquia e atendimento individual, ${ }^{18}$ além de aumentar o trabalho para as equipes de referência e necessidade do cumprimento de metas. ${ }^{15}$ Outros fatores apontados como dificultadores
ISSN 2236 - 1987

foram: a transferência de responsabilidade para o apoiador ${ }^{17}$ ou disputa para saber de quem é a responsabilidade, ${ }^{14} \quad 0 \quad$ que consequentemente fragmenta o trabalho, uma vez que não são estabelecidos vínculos profissionais e partilha de saber entre equipes.

Torna-se necessário buscar mecanismos que minimizem os fatores dificultadores, tendo em vista que os mesmos enfraquecem a potência transformadora do AM, assim como limita 0 alcance do seu objetivo enquanto prática que visa apoiar e qualificar o trabalho na atenção básica, possibilitando maior resolutividade e integralidade das ações.

Foi possível constatar que a maioria dos estudos apontaram fatores dificultadores. Esse dado chama a atenção para a possibilidade do "não sucesso" do AM, assim como para a possibilidade de reverter tais dificuldades em objetivos a serem superados com a busca do estabelecimento de interação e matriciamento mais estruturados e efetivos.

\section{CONSIDERAÇÕES FINAIS}

0 estudo possibilitou compreender os diferentes processos de matriciamento existentes no âmbito da APS. Os relatos dos profissionais revelam as potencialidades e o enriquecimento para o processo de trabalho, proporcionado pelo apoio, refletindo na melhoria do cuidado ofertado e organização do sistema. 0 vínculo e envolvimento profissional com a modalidade de trabalho foram apontados como fatores essenciais 


\section{JOURNALF \\ ISSN $2236-1987$}

para o sucesso de tal modo de fazer saúde.

Porém percebe-se ainda aspectos dificultadores para a efetivação do matriciamento, ora relacionados aos profissionais como o desinteresse, falta de conhecimento, resistência e diferentes entendimentos sobre 0 processo de trabalho, ora relacionados ao serviço, como a rotatividade profissional, escassez de carga horária e excesso de atribuições.

Apesar dos desafios ainda presentes para seu total sucesso, os resultados apontam para o auxílio ofertado pelo $A M$ no que diz respeito ao gerenciamento do trabalho na APS. Uma limitação de generalização do estudo é de que a revisão se baseou apenas em artigos de acesso gratuito, o que pode ter deixado de captar outras publicações que pudessem abordar a temática da revisão.

Os resultados obtidos evidenciam a relevância da inserção de práticas do $A M$ às equipes de APS a fim de aprimorar o cuidado ofertado na busca pela integralidade da assistência, assim como para os reflexos positivos que essa ação desencadeia para a gestão desses serviços. Sugere-se o desenvolvimento de estratégias que visem à implantação e o fortalecimento do $A M$ no âmbito dos cuidados primários em saúde. Recomenda-se também maior investimento em publicações de tais ações, visando o embasamento prático.

\section{REFERÊNCIAS}

1 Ministério da Saúde (BR). Secretaria de Atenção à Saúde. Política Nacional de Humanização da Atenção e Gestão do SUS. Clínica ampliada e compartilhada [Internet]. Brasília; 2009[acesso em 2019 ago 26]. Disponível em: http://bvsms.saude.gov.br/bvs/public acoes/clinica_ampliada_compartilhad a.pdf

2 Cunha GT, Campos GWS. Apoio matricial e atenção primária em saúde. Saúde Soc. [Internet]. 2011 jul[acesso em 2018 nov 12];20(4):961-70. Disponível em: http:/ / www. scielo.br/pdf/sausoc/v20 n4/13.pdf

3 Campos GWS, Figueiredo MD, Pereira Junior N, Castro CP de. A aplicação da metodologia paideia no apoio institucional, no apoio matricial e na clínica ampliada. Interface comum. saude educ. [Internet]. 2014[acesso em 2018 nov 12];18(1):983-95. Disponível em: http://www.scielo.br/pdf/icse/v18s1 /1807-5762-icse-18-1-0983.pdf

4 Jorge MSB, Vasconcelos MGF, Neto JPM, Gondim LGF, Simões ECP. Possibilidades e desafios do apoio matricial na atenção básica: percepções dos profissionais. Psicol. teor. prát. [Internet]. 2014[acesso em 2018 nov 12];16(2):63-74 Disponível em:

http://pepsic.bvsalud.org/pdf/ptp/v1 6n2/06.pdf

5 Ministério da Saúde (BR). Portaria $n^{\circ}$ 154 , de 24 de janeiro de 2008. Cria os Núcleos de Apoio à Saúde da Família NASF [Internet]. Brasília; 2008[acesso em 2019 ago 26] Disponível em: http: / /bvsms.saude.gov.br/bvs/saude legis/gm/2008/prt0154_24_01_2008.h tml 


\section{JOURNALOF \\ NURSING \\ ANO HEALTH}

ISSN 2236 - 1987

Disponível em: http://www.scielo.br/pdf/csc/v18n7/ 31.pdf

12 Freire FMS, Pichelli AAWS. 0 psicólogo apoiador matricial: percepções e práticas na Atenção Básica. Psicol. ciênc. prof. [Internet]. 2013[acesso em 2018 nov12];33(1):162-73. Disponível em: http://www.scielo.br/pdf/pcp/v33n1 /v33n1a13.pdf

13 Sampaio J, Martiniano CS, Rocha AMO, Souza Neto AA de, Sobrinho GDO, Marcolino EC, et al. Núcleo de apoio à saúde da família: refletindo sobre as acepções emergentes da prática. Rev. Bras. Ciênc. Saúde (João Pessoa, Online). [Internet]. 2013[acesso em 2018 nov 12];17(1):47-54. Disponível em:

http://www.periodicos.ufpb.br/ojs/in dex.php/rbcs/article/view/15932/940 4

14 Klein AP, D'Oliveira AFPL. O "cabo de força" da assistência: concepção e prática de psicólogos sobre o apoio matricial no núcleo de apoio à Saúde da Família. Cad. Saúde Pública (Online) [Internet]. 2017[acesso em 2018 nov 12];33(1):e00158815. Disponível em: http://www.scielo.br/pdf/csp/v33n1 /1678-4464-csp-33-01-e00158815.pdf

15 Matuda CG, Pinto NRS, Martins CL, Frazão P. Colaboração interprofissional na estratégia saúde da família: implicações para a produção do cuidado e a gestão do trabalho. Ciênc. Saúde Colet. [Internet]. 2015[acesso em 2018 nov 12];20(8):2511-21. Disponível em: http://www.scielo.br/pdf/csc/v20n8/ 1413-8123-csc-20-08-2511.pdf 


\section{NURSING \\ ${ }^{\text {ANO HEALTH }}$}

16 Tatmatsu DB, Araújo ACC. Atenção primária e saúde mental: contribuições e potencialidades do apoio matricial. Mudanças. [Internet]. 2016 jul/dez[acesso em 2018 nov 12];24(2):71-9. Disponível em: https://www.metodista.br/revistas/r evistas-

ims/index.php/MUD/article/view/672 $0 / 5542$

17 Sampaio J, Melo MC de, Grigório MC, Soares RS. Processos de trabalho dos núcleos de atenção à saúde da família junto a atenção básica: implicações para a articulação de redes territoriais de cuidados em saúde. Rev. bras. ciênc. saúde [Internet]. 2015 jan[acesso em 2018 nov 12];19(1):41 $8 . \quad$ Disponível em: http://www.periodicos.ufpb.br/ojs/in dex.php/rbcs/article/view/20163/150 47

18 Iglesias A, Avellar LZ. As contribuições dos psicólogos para o matriciamento em saúde mental. Psicol. ciênc. prof. [Internet]. 2016 abr/jun[acesso em 2018 nov 12];36(2):364-79. Disponível em: http://www.scielo.br/pdf/pcp/v36n2 /1982-3703-pcp-36-2-0364.pdf

19 Hoepfner C, Franco SC, Maciel RA, Hoepfner AMS. Programa de apoio matricial em cardiologia: qualificação e diálogo com profissionais da atenção primária. Saúde Soc. [Internet]. 2014[acesso em 2018 nov 12];23(3):1091-101. Disponível em: http: / / www.scielo.br/pdf/sausoc/v23 n3/0104-1290-sausoc-23-3-1091.pdf

20 Fittipaldi ALM, Romano VF, Barros DC de. Nas entrelinhas do olhar: apoio matricial e os profissionais da estratégia saúde da família. Saúde
ISSN 2236 - 1987

debate. [Internet]. 2015 jan[acesso em 2018 nov 12];39(104):76-87. Disponível em:

http: / /www.scielo.br/pdf/sdeb/v39n 104/0103-1104-sdeb-39-104-00076.pdf

21 Garcia GDV, Silva IF da, Cavalcante M, Cervo MR, Zambenedetti G, ZanotiJeronymo DV. Apoio matricial na atenção à saúde mental em uma regional de saúde, Paraná, Brasil. Saude e pesqui. (Impr.). [Internet]. 2017 set/dez[acesso em 2018 nov 12];10(3):423-32. Disponível em: http: / / periodicos.unicesumar.edu.br/ index.php/saudpesq/article/view/585 4/3125

Data de submissão: $21 / 10 / 2018$

Data de aceite: 17/06/2019

Data de publicação: 18/09/2019 